\title{
Pratiques
}

Linguistique, littérature, didactique

179-180 | 2018

Poésie et langue : aspects théoriques et didactiques

\section{Faire sens et avoir un sens}

Note sur quelques aspects de la signifiance linguistique

Note on some aspects of lingusitic's significance

\section{Régis Missire}

\section{(2) OpenEdition}

Journals

Édition électronique

URL : http://journals.openedition.org/pratiques/4830

DOI : 10.4000/pratiques.4830

ISSN : 2425-2042

Éditeur

Centre de recherche sur les médiations (CREM)

Référence électronique

Régis Missire, «Faire sens et avoir un sens », Pratiques [En ligne], 179-180 | 2018, mis en ligne le 31 décembre 2018, consulté le 19 avril 2019. URL : http://journals.openedition.org/pratiques/4830 ; DOI : 10.4000/pratiques.4830

Ce document a été généré automatiquement le 19 avril 2019.

(c) Tous droits réservés 


\title{
Faire sens et avoir un sens
}

\author{
Note sur quelques aspects de la signifiance linguistique \\ Note on some aspects of lingusitic's significance
}

Régis Missire

je n'ai plus à chercher

à comprendre

puisque je suis

moi-même

l'inconnu qui arrive

au sens

H. Meschonnic (2006, p. 49)

1 La distinction établie par É. Benveniste (1974) entre modes sémiotique et sémantique trace encore aujourd'hui une ligne de partage entre deux types d'approche dans les linguistiques du discours d'ascendance saussurienne: on identifie ainsi d'une part une tradition post-hjelmslevienne qui a étendu la problématique sémiotique à des unités empiriques étendues (comme les textes), d'autre part une tradition post-benvenistienne pour laquelle l'impossibilité d'une telle extension a justifié le développement d'un concept de signifiance problématisant à nouveaux frais les relations sons/sens dans une linguistique de la parole. Si la première, concrétisée par plusieurs théories sémantiques, a privilégié la description du plan du signifié linguistique, le lien plus serré que la seconde a maintenu avec le signifiant linguistique l'a amenée à retrouver des phénomènes auparavant décrits par la stylistique et la poétique. Nous souhaitons dans ce travail contribuer à l'éclairage de certains aspects de ce partage des objets et des disciplines, en prenant comme point d'entrée la notion de signifiance telle qu'elle a été élaborée depuis les années 1960 en linguistique, en faisant l'hypothèse que, en dépit de la diversité des appropriations qui ont pu en être faites, cette notion solidarise un ensemble de phénomènes fondamentaux pour l'étude du langage poétique.

2 Définissable en première approximation comme « le fait pour une unité de signifier $»^{1}$, la notion de signifiance est liée morphologiquement ${ }^{2}$, logiquement ${ }^{3}$ et historiquement à celle de signifiant. Sur le plan historique en particulier, la notion de signifiance a été portée dans les années 1960-70 par des auteurs diversement influencés par la «logique du 
signifiant » lacanienne ${ }^{4}$, dont la diffusion avait débuté une quinzaine d'années plus tôt, et en réaction à certaines approches structurales, qui développaient au même moment sous le nom de «sémiotique » une sémantique «idéaliste » ne conférant au signifiant qu'un rôle ancillaire ${ }^{5}$.

Le propos se veut de repérage et de synthèse : l'enjeu est de parvenir à dégager des points d'articulation entre considérations linguistiques et réflexions sur la signifiance issues des théories du texte, en identifiant quelques thèmes récurrents et en formulant des distinctions.

Nous procédons en cinq temps : la première partie traite du couple signifiance/signification à la lumière de la distinction activité/produit dans le domaine langagier. La deuxième déplace légèrement le point de vue dans la perspective de l'axe actuel/virtuel. La troisième introduit, sur la base des deux discussions précédentes, une distinction entre trois soustypes de signifiance, qui sont brièvement illustrées dans la quatrième partie. Nous proposons enfin en conclusion une discussion sur l'opposition benvenistienne du sémantique et du sémiotique ainsi que sa lecture meschonicienne.

\section{Signifiance et signification : faire sens vs avoir un sens}

Indépendamment des spécificités qu'a connues l'appropriation du concept de signifiance ces cinquante dernières années, une constante dans son usage a consisté dans l'intention de ses promoteurs de restituer la dimension processuelle de l'activité langagière, qu'une approche seulement guidée par le concept de signification échoue à saisir parce qu'elle ne vise que l'état final du processus d'assignation de sens, le résultat stabilisé en lequel se résorbe et s'annule in fine l'activité du locuteur. Entre signification et signifiance, il y a alors le même écart qu'entre le langage envisagé en tant qu'érgon d'une part et enérgeia de l'autre (Coseriu, 2001), ou, dans les termes plus matérialistes de R. Barthes (1974, p. 4-6) dans son article de synthèse sur la théorie du texte, le même écart qu'entre produit et production:

On peut attribuer à un texte une signification unique et en quelque sorte canonique; [...] on traite le texte comme s'il était dépositaire d'une signification objective, et cette signification apparaît comme embaumée dans l'œuvre-produit. Mais dès lors que le texte est conçu comme une production (et non plus comme un produit), la « signification » n'est plus un concept adéquat. [...] il devient nécessaire de bien distinguer la signification, qui appartient au plan du produit, de l'énoncé, de la communication, et le travail signifiant, qui, lui, appartient au plan de la production, de l'énonciation, de la symbolisation: c'est ce travail qu'on appelle la signifiance. La signifiance est un procès, au cours duquel le «sujet» du texte, échappant à la logique de l'ego-cogito et s'engageant dans d'autres logiques (celle du signifiant et celle de la contradiction), se débat avec le sens et se déconstruit («se perd»); la signifiance, et c'est ce qui la distingue immédiatement de la signification, est donc un travail.

Dans ce tableau, il faudrait ainsi choisir entre approches philologico-herméneutiques d'une part, auxquelles reviendraient l'arpentage des espaces textuels et l'établissement du cadastre des significations, et la "sémanalyse» d'autre part (Kristeva, 1969), qui prendrait en charge l'étude du travail de la signifiance. Il faut bien sûr resituer cette citation dans le contexte "post-structuraliste » de l'époque et sa défiance envers un structuralisme $^{6}$ dont les théorisations étaient critiquées (notamment) pour leur inaptitude à rendre compte de l'activité du sujet dans la langue. Plutôt que l'avoir un sens, 
c'est donc le faire sens qui est au centre de l'attention, en affinité par exemple avec les réflexions contemporaines d'É. Benveniste sur le langage poétique de C. Baudelaire ( $« \mathrm{La}$ langue poétique et plus précisément la poétique ne consiste pas à dire, mais à faire.» [Benveniste, 2011, p. 400]), défendant dans une note intitulée "différences d'approche » une telle position contre l'approche «objectale » de C. Lévi-Strauss et R. Jakobson dans leur analyse du poème Les Chats (et qui pourrait valoir aussi bien pour d'autres études de sémantique structurale qui lui étaient contemporaines):

Une approche consiste à partir de la pièce de vers comme d'une donnée, de la décrire, de la démonter comme un objet. C'est l'analyse telle qu'on la trouve appliquée aux Chats dans le bel article de Lévi-Strauss et Jakobson. Une autre approche sera d'un type tout autre. On s'efforcera d'atteindre la structure profonde de son univers poétique dans le choix révélateur des images et dans leur articulation. (Benveniste, 2011, p. 186)

$7 \quad$ Afin d'éviter le psychologisme qu'un tel souci du sujet et du faire pourrait susciter, il est fréquent que les formulations des théoriciens de la signifiance neutralisent l'opposition des pôles énonciatifs et réceptifs (toute interprétation étant in fine conçue comme réénonciation), les propriétés de l'activité linguistique des locuteurs pouvant alors par métonymie être prédiquées directement du texte, dont on dira par exemple qu'il «travaille $»^{7}$, ou de ses unités, comme dans cette définition de manuel : «[...] signifiance organisation des chaînes prosodiques produisant une activité des mots qui ne se confond pas avec leur sens mais participe de leur force, indépendamment de toute conscience qu'on peut en avoir ${ }^{8}$.» (Dessons \& Meschonnic, 1998, p. 44, nous soulignons). L'analyse s'efforcera alors, en amont de toute fixation de sens, corrélative d'un arrêt du parcours interprétatif, de saisir l'enérgeia langagière, avec ce que cela implique d'indétermination et d'ouverture sémantique. On peut représenter simplement cette première différence entre les deux problématiques de la signification et de la signifiance.

Figure 1. Relations de signification et de signifiance

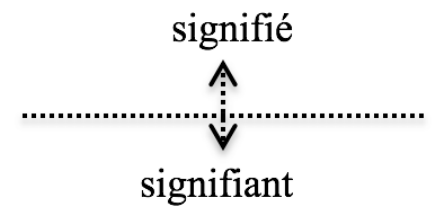

Relation de signification

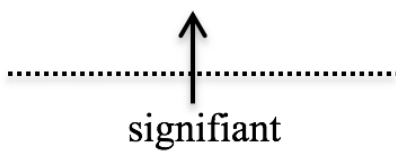

Relation de signifiance

Dans la relation de signification, la double flèche figure la relation de présupposition réciproque entre signifiant et signifié telle que l'a théorisée la descendance hjelmslevienne de F. de Saussure avec le concept de sémiosis (cf. par exemple Greimas \& Courtès, 1993), relation «enveloppée » par les termes qu'elle relie; dans la relation de signifiance, le caractère plein de la flèche simple représente au contraire la prééminence de la relation et d'un de ses termes (le signifiant), sur le terme aboutissant de la relation, qui reste une simple position vide, mais nécessaire. 


\section{Signification et signifiance : actuel déterminé vs virtuel indéterminé}

9 Le schéma précédent laisse apparaitre une seconde distinction entre signification et signifiance : dans la relation de signification, le signifié, puisqu'il hérite immédiatement (par présupposition réciproque) du mode d'existence du signifiant, est par définition actuel, alors qu'il est seulement potentiel dans la relation de signifiance, à tout le moins retardé dans son actualisation. L'expérience de la relation de signification est celle d'une conversion immédiate entre les pôles de la dualité forme-sens qu'est le signe linguistique (Saussure, 2002), alors que celle de la signifiance réside au contraire dans une rétention installant le sujet sur le vecteur sémiotique de la flèche signifiante et différant l'actualisation de son terme ad quem - que cette rétention procède d'une pratique délibérée (plaisir du texte, jouissance du signifiant...) ou non (effet retard du sens). Cette différence entre régimes sémiotiques ${ }^{9}$ au niveau textuel (i.e. au niveau de la parole concrète) trouve un corrélat au niveau linguistique le plus fondamental. On peut en effet reprendre dans cette perspective la distinction établie par É. Benveniste entre modes sémiotique et

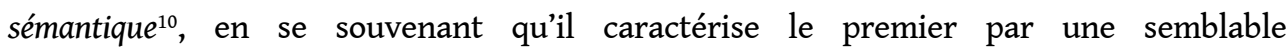
indétermination du sens : « Dans la langue organisée en signes, le sens d'une unité est le fait qu'elle a un sens, qu'elle est signifiante. Ce qui équivaut à l'identifier par sa capacité de remplir une fonction propositionnelle. C'est la condition nécessaire et suffisante pour que nous reconnaissions cette unité comme signifiante. [...] Un tout autre problème serait de se demander: quel est ce sens? » (Benveniste, 1966, p. 127, nous soulignons), ou encore «En sémiologie, ce que le signe signifie n'a pas à être défini [...], signifier c'est avoir un sens, sans plus [...] Le signe a toujours et seulement valeur générique et conceptuelle. Il n'admet donc pas de signifié particulier ou occasionnel.» (Benveniste, 1974, p. 222, nous soulignons), conception dans laquelle on retrouve la relation de signifiance schématisée ci-dessus. En somme, le régime de la signifiance, entendu comme fléchage sémiotique qui, sans pointer un signifié particulier, modalise le signifiant comme "signifiant", est la relation sémiolinguistique fondamentale, qui permet par exemple d'expliquer que la perception du langage diffère de celle d'autres stimuli acoustiques ou graphiques. La concevoir ainsi offre également une alternative à la description de la relation entre signifiant et signifié sur le seul modèle associationniste, qui échoue à rendre compte du fait que c'est précisément cette modalisation du formant linguistique qui le transforme en signifiant.

\section{Système, normes, textes : trois niveaux de la signifiance}

10 Pour nécessaire qu'apparaisse la distinction benvenistienne d'une double signifiance, la première (sémiotique) concernant le niveau abstrait des signes d'un système linguistique, la seconde (sémantique) celle de leur concrétisation dans le discours, elle demeure insuffisante pour saisir clairement que la transition de la langue à la parole ne consiste pas seulement dans celle des unités (abstraites) d'un système à leur concaténation dans la phrase (concrète). E. Coseriu (1952) a en effet montré que l'opposition saussurienne de la langue et la parole reçoit dans le $C L G$ des valeurs très variables, relevant de trois grandes oppositions (virtuel/actuel [ou système/réalisation], abstrait/concret et collectif/individuel), ce 
qui l'amène à remplacer l'opposition binaire langue/parole par une opposition ternaire entre système, norme, et parler concret :

1) Si l'opposition s'établit entre système et réalisation, la langue correspond uniquement au système, et la parole à tous les autres concepts incluant les divers degrés d'abstraction (normes sociales et individuelles) et le plan concret du " parler». 2) Si l'opposition s'établit entre concret et abstrait, la parole coïncide avec le "parler ", et la langue correspond à tous les autres concepts incluant les divers degrés d'abstraction (normes et système), qui, cependant, se manifestent concrètement dans le «parler». 3) Si l'opposition s'établit entre social et individuel, la langue comprend le système et la norme, et la parole inclut la norme individuelle et le "parler " concret, qui contiennent cependant les deux autres niveaux. (Coseriu, 1952, p. 101, notre traduction).

Nous avons proposé (Missire, 2018b) de représenter ainsi cette tripartition :

Tableau 1. Niveaux de l'analyse linguistique

\begin{tabular}{|c|c|c|c|c|}
\hline & \multicolumn{4}{|c|}{ niveaux de l'analyse } \\
\hline & \multicolumn{3}{|l|}{ abstrait } & \multirow[t]{2}{*}{ concret } \\
\hline & virtuel & actuel & & \\
\hline & \multicolumn{2}{|l|}{ social } & \multicolumn{2}{|l|}{ individuel } \\
\hline typologie d'E. Coseriu & système & normes sociales & normes individuelles & parler concret \\
\hline \multirow{3}{*}{ typologies de F. de Saussure } & langue & parole & parole & parole \\
\hline & langue & langue & langue & parole \\
\hline & langue & langue & parole & parole \\
\hline
\end{tabular}

Dans une telle conception, le système correspond à l'ensemble des unités phonomorphologiques d'une langue ainsi qu'à leurs règles de combinaison, indépendamment $d u$ fait que telle combinaison soit attestée ou non: la productivité morphologique, les mécanismes analogiques sous-tendant les innovations néologiques, constructionnelles, syntaxiques ${ }^{11}$ en donnent une bonne image. Au niveau des normes se situent au contraire uniquement les unités attestées qui auront été actualisées dans la parole. Pour illustrer cette différence entre système et normes, E. Coseriu (2001, p. 247) prend l'exemple d'un adjectif français : «Il y a quelques années, on pouvait dire (et nous l'avons entendu) : "le terme notionnel n'existe pas en français, il ne figure pas dans le Larousse" [...] Mais ce n'était vrai qu'au point de vue de la norme du français ; dans le système, le terme notionnel était virtuellement existant ( possible $)^{12}$. " De ce point de vue, la plupart des unités lexicales d'une langue sont déjà des unités du niveau des normes. Si système et normes s'opposent donc selon la distinction virtuel/actuel, ils ont cependant en commun d'être des abstractions construites à partir des occurrences concrètes de parole que sont les textes ${ }^{13}$. C'est alors l'opposition type/occurrences qui est pertinente : les unités de système et de normes sont des types construits tant dans les pratiques métalinguistiques du linguiste qu'épilinguistiques des locuteurs. En gardant à l'esprit ces distinctions, on comprend alors en quoi la représentation de la parole comme seule syntagmation d'unités empêche 
aussi bien de distinguer des types d'unités syntagmatiques (donc des unités actuelles abstraites) que des concrétisations de signes de systèmes (donc des unités virtuelles concrètes, par exemple la valeur que peut recevoir tel morphème dans un texte particulier dès lors que l'interprétation « focalise » sur lui ${ }^{14}$ ).

Mettons alors en relation le couple signification/signifiance avec ces distinctions conceptuelles. La difficulté est ici liée au fait qu'à chacun des trois niveaux sont situées des unités doubles (signifiant et signifié), et que le statut de chacune de leur face varie en fonction du niveau considéré. Une première approximation pourrait nous amener à indexer la signifiance au niveau du système (les morphèmes signifient, mais sont avant tout reconnus par les locuteurs comme signifiants, sans qu'ils puissent leur assigner de signifié déterminé) et la signification au niveau des normes (cf. par exemple les acceptions lexicales); quant aux régimes de la désignation et de l'expression, ils relèveraient uniquement de la parole concrète, où se déterminent la référence et l'intenté du discours, qu'É. Benveniste indexait dans le domaine sémantique.

Tableau 2. Signification, signifiance et niveaux de l'analyse 1

\begin{tabular}{|c|c|c|c|}
\hline & \multicolumn{2}{|l|}{ abstrait } & \multirow[t]{2}{*}{ concret } \\
\hline & virtuel & actuel & \\
\hline instances & système & normes & textes \\
\hline régimes sémiotiques & signifiance & signification & $\begin{array}{l}\text { expression } \\
\text { désignation }\end{array}$ \\
\hline modes (É. Benveniste) & sémiotique & sémiotique & sémantique \\
\hline
\end{tabular}

Mais on voit le problème que pose un tel tableau : on ne peut pas seulement corréler ces quatre régimes sémiotiques à des niveaux de l'analyse linguistique abstraitement distingués, pour la raison essentielle que ceux-ci coexistent concrètement dans l'activité de parole, au sein de laquelle ils sont toujours discernables au titre de phases de cette activité. Il faut insister sur ce point, signalé déjà par E. Coseriu (2001, p. 31-36, nous soulignons):

le langage n'existe concrètement que comme activité, comme activité de parler [...] et ce n'est que parce que le langage se manifeste comme une activité qu'on peut l'étudier aussi en tant que "produit" [...] étudier la langue, c'est étudier une dimension de l'activité de parler, dimension qui n'est ni abstraite ni extérieure à cette activité. [...] la langue est contenue dans la parole et la distinction langueparole - en dehors du fait qu'elle peut être interprétée de différentes façons - n'est pas « réelle », mais bien « formelle » et méthodologique.

Cela signifie qu'au niveau de l'activité langagière concrète, c'est-à-dire au niveau des textes envisagés comme enérgeia, il faut prévoir la co-existence des quatre régimes de la signifiance, de la signification, de l'expression et de la désignation, tout comme il faut, dès le niveau des normes, prévoir celle des régimes de la signifiance et de la signification (expression et désignation en étant en revanche exclus). La notion générale de signifiance peut alors trouver à se spécifier selon les lieux où on la considère : 
16 (i) Au niveau du système, la signifiance correspond, comme on l'a évoqué supra, à la modalisation d'un formant linguistique identifié comme signifiant, sans que l'on ait à se préoccuper des sens qu'il peut recevoir, à supposer que ceux-ci soient déterminables. C'est le niveau sémiotique tel qu'il est caractérisé par É. Benveniste (1974, p. 222): «L'entité considérée signifie-t-elle? La réponse est oui, ou non. Si c'est oui, tout est dit, on l'enregistre; si c'est non, on la rejette, et tout est dit aussi. » Nous dénommerons conventionnellement signifiance 1 ce type, fondamental, de signifiance.

17 (ii) Au niveau des normes, s'oppose au caractère virtuel de la relation de signifiance celui actuel de la relation de signification, qui actualise les différents signifiés associés à une forme, relation dont l'immédiateté ${ }^{15}$ " enveloppe » la relation signifiance 1, qui y reste cependant présente et remobilisable à tout moment: l'alternative d'É. Benveniste évoquée ci-dessus ( «l'entité considérée signifie-telle ?...») peut d'ailleurs s'appliquer à des unités du niveau des normes. Mais on observe également à ce niveau une spécification de la relation de signifiance: parce que le formant lexical ouvre sur le réseau des différents signifiés associés à l'unité (réseau de signifiés qui constitue la valeur de l'unité dans une langue donnée), la potentialité de la relation de signifiance se précise dans la capacité d'évocation de ces différentes valeurs par le signifiant, soutenant, selon la belle formule de R. Barthes (1974, p. 7, nous soulignons), un "arbre d'associations» sémantiques : «la signifiance [...] est indistinctement à tous les niveaux de l'œuvre [...] dans les monèmes, qui sont moins des unités sémantiques que des arbres d'associations et sont entraînés par la connotation, la polysémie latente, dans une métonymie généralisée. » Nous dénommerons conventionnellement signifiance 2 cette spécification de la relation de signifiance.

(iii) Au niveau de la parole concrète enfin, la spécificité de la relation de signifiance réside dans les effets co-textuels du chainage syntagmatique des signifiants, qui crée, pour reprendre les termes d'H. Meschonnic (1982, p. 217), une "sémantique spécifique » en déterminant les relations de signification et de signifiance 1 et $2^{16}$ : classiquement, une assonnance ou une allitération pourront susciter une syllepse dans l'interprétation d'un texte poétique, mais cet effet sémantique n'a cependant rien de systématique. Nous dénommerons signifiance 3 ce troisième type.

Ce qui justifie le rapprochement de ces trois types de relation sous le terme générique de signifiance réside dans leur caractère potentiel et indéterminé: indétermination sémantique de signifiance 1 , évocation sémantique rhizomatique de signifiance 2 , détermination de ces deux premières relations par signifiance 3 , dans un texte dont l'interprétation est toujours un événement singulier. Ainsi un phénomène d'isophonie pourra aussi bien selon les situations :

20 (i) être remarqué pour lui-même dans un moment figural (Jenny, 1990) de l'interprétation, sans pour autant engager de mise en série des unités concernées par le phénomène (signifiance 3 seule);

21 (ii) susciter une telle sériation des unités lexicales et les interactions sémantiques afférentes entre leurs signifiés, voire engager des effets plus radicaux de type sylleptique (signifiance 3 et signifiance 2);

22 (iii) aboutir à une sémantisation (souvent de type iconique) des constituants phonétiques concernés (signifiance 3 , signifiance 1 , et relation de signification, celle-ci sanctionnant l'aboutissement du parcours interprétatif). 
NB : Nous prenons l'exemple de l'isophonie et de ses effets sur la signification lexicale par commodité. Mais les phénomènes de signifiance ne s'y limitent évidemment pas, et concernent tous les niveaux de l'analyse linguistique. Ainsi par exemple, les phénomènes d'enjambement, d'absolutisation à la césure, etc. produisent-ils des effets tout à fait comparables sur le plan de signifiance 2. En guise d'illustration, le lecteur pourra mesurer l'effectivité de tels moyens en étudiant comment le retour à la ligne dans le poème d'H. Meschonnic (2006) en épigraphe induit le dédoublement des structures dans une sorte de « double détente » syntaxique presque systématique.

Notre tableau initial doit donc être modifié ainsi.

Tableau 3. Signification, signifiance et niveaux de l'analyse 2

\begin{tabular}{|c|c|c|c|}
\hline & \multicolumn{3}{|l|}{ abstrait } \\
\hline & virtuel & actuel & \\
\hline instances & système & normes & textes \\
\hline régimes sémiotiques & signifiance 1 & $\begin{array}{l}\text { signifiance } 1 \text { et } 2 \\
\text { signification }\end{array}$ & $\begin{array}{l}\text { signifiance } 1,2 \text {, et } \\
3 \\
\text { signification } \\
\text { expression } \\
\text { désignation }\end{array}$ \\
\hline modes (É. Benveniste) & sémiotique & sémiotique & sémantique \\
\hline
\end{tabular}

\section{Illustration}

Illustrons quelques modalités de mises en relation entre signifiance et signification ${ }^{17}$ en considérant le premier quatrain de Mon rêve familier (P. Verlaine) :

\footnotetext{
Je fais souvent ce rêve étrange et pénétrant

D'une femme inconnue, et que j'aime et qui m'aime

Et qui n'est, chaque fois, ni tout à fait la même

Ni tout à fait une autre, et m'aime et me comprend.
}

Nous procédons en rubriquant par commodité nos remarques selon les distinctions introduites précédemment (signifiance 1, 2 et 3, signification). Insistons encore: tous les phénomènes désignés par ces concepts étant toujours présents, le rubriquage ne fait que signaler des dominantes, sur lesquelles l'accent est mis pour des raisons d'illustration.

\section{(i) Signifiance $3 \rightarrow$ signifiance 1}

Le dernier hémistiche du quatrième vers présente une condensation ${ }^{18}$ des valeurs phoniques les plus denses du quatrain, elles-mêmes sources de plusieurs mises en séries lexématiques dans les trois premiers vers, qui produit un effet de clausule à la fin du quatrain, l'effet de résonnance paronomastique étant renforcé par cette condensation. L'effet, figural, s'apprécie avant tout dans le différentiel diffus/compact entre espaces 
textuels, sans pour autant qu'aucun prétendant à signifiance 2 n'émerge. Le passage concerné est potentialisé ${ }^{19}$ par le phénomène induisant une présomption de sens (signifiance 1), sans que, nous semble-t-il, celle-ci puisse ici se résoudre dans une relation de signification affectant une unité sémio-linguistique particulière.

Figure 2. Signifiance 3 /signifiance 1 - condensation de signifiants

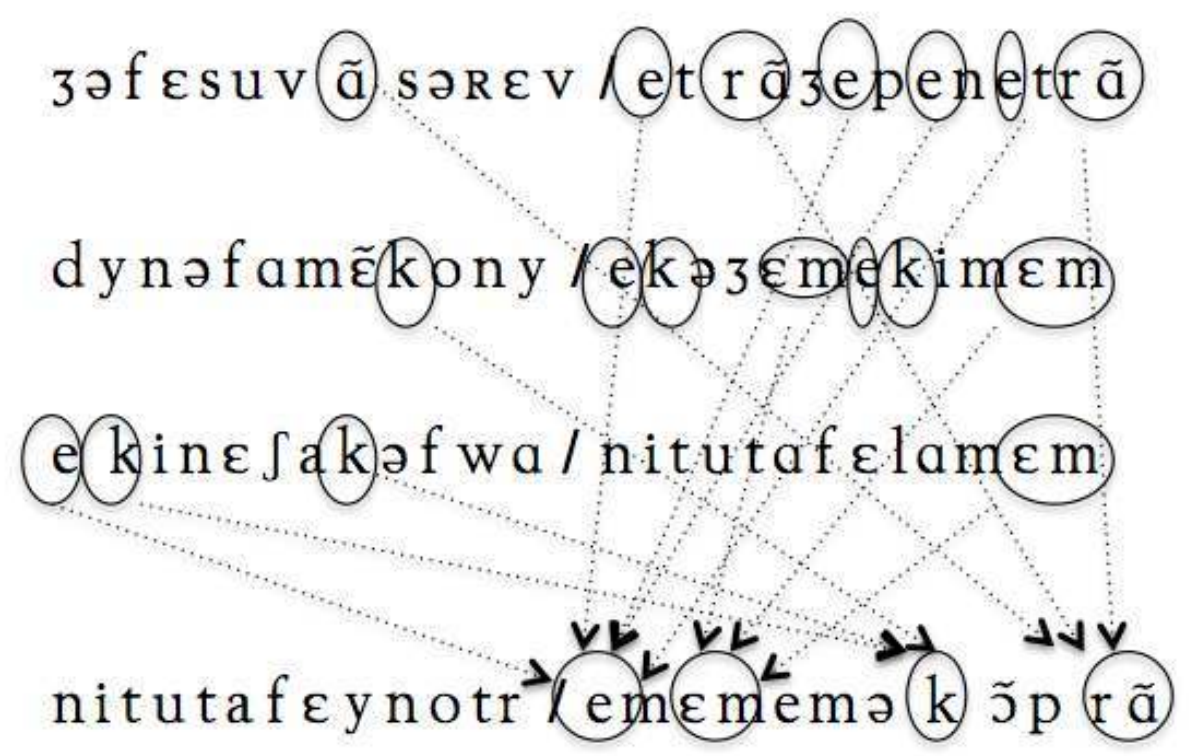

\section{(ii) Signifiance $3 \rightarrow$ signifiance 2}

Considérons plus avant la série "étrange ", "pénétrant », "comprend » créée par la répétition de [Rõ]. Aucune raison d'attribuer une valeur sémantique directe au groupe de phonèmes support du phénomène de répétition, et on suit ici H. Meschonnic (1973, p. 307, nous soulignons) : «[...] par la grille des phonèmes, ce n'est pas les phonèmes qui font signe, c'est les mots par et dans leur matérialité signifiante : on n'attribue pas aux phonèmes la valeur des mots». Mais il faut donc décrire au niveau du sémantisme lexical les effets produits par cette signifiance $3:$ les signifiés respectifs des unités se voient en effet affectés par la sériation, le rapprochement de «étrange » et " pénétrant " produisant un écho sémantique inversé entre l'/extériorité/ du premier et l' /intériorisation/ du second ${ }^{20}$. Pris dans la série, « comprend » se trouve alors enrolé dans la même isotopie spatiale, et neutralise l'opposition /intériorité/-/extériorité/ par actualisation de la valeur / inclusion/. On a affaire ici typiquement à un phénomène de signifiance 2 : alors que le contexte proche de « comprend " préactivait l'acception cognitive de " comprendre », la relation à distance via la paronomase prescrit l'actualisation de l'acception spatiale, avec au final un battement sylleptique sur ce dernier mot du quatrain. 


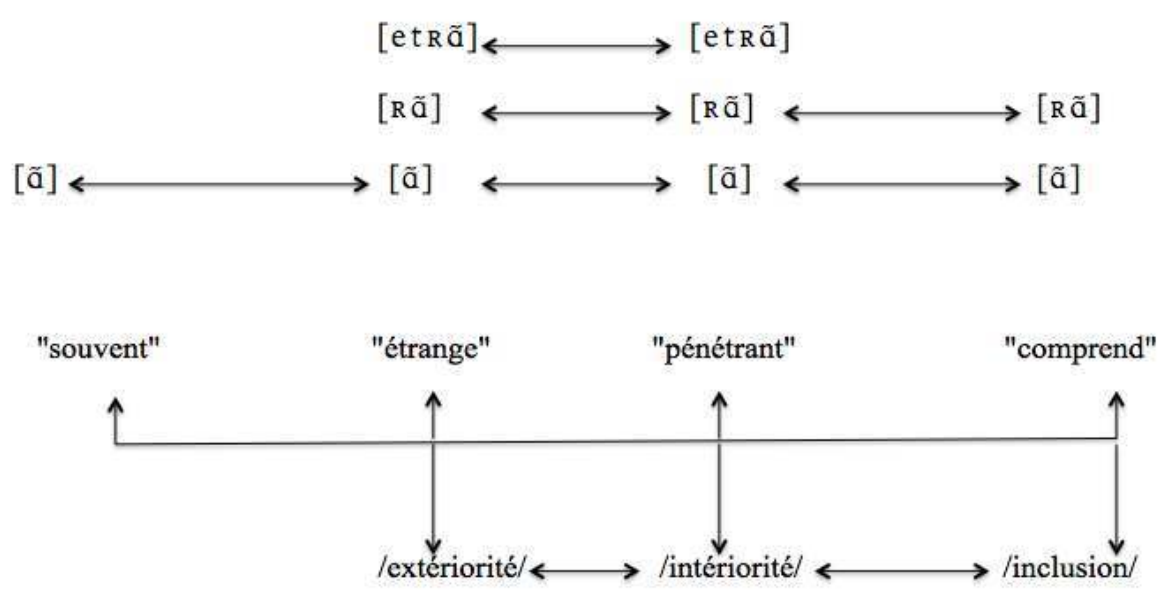

\section{(iii) Signifiance $3 \rightarrow$ signifiance $1 \rightarrow$ signification}

Dans les deuxième et troisième vers, on note la récurrence à cinq reprises d'une matrice consonantique nécessitant pour être décrite la mobilisation de critères syllabiques, phonétiques, et accentuels. La matrice :

(i) est trisyllabique,

(ii) contient un $[\mathrm{k}]$ sur la deuxième position,

(iii) est accentuée sur la troisième position (nous marquons l'accent de groupe par un soulignement) :

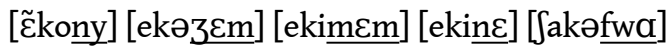

Contrairement à l'exemple précédent, les faisceaux de répétitions phoniques ne permettent pas de créer une série avec des lexèmes nettement identifiés. Cependant, l'identification de cet effet de signifiant, et l'éventuelle perception de la forme phoniqueaccentuelle peut, dans ce contexte, faire l'objet d'une sémiotisation (relation de signification $)^{21}$ : on peut ainsi indexer la répétition de cette forme sur l'isotopie de la / familiarité/, présente dans le début du poème. On considère alors que la répétition de la forme contribue à l'isotopie, agissant linguistiquement ce que signifient lexicalement certains unités du texte («familier », « souvent », " chaque fois », le présent d'habitude). Le phénomène est comparable à celui présent dans le vers célèbre de $\mathrm{C}$. Baudelaire Comme de longs échos qui de loin se confondent, avec d'une part la répétition des nasales (Benveniste, 2011, p. 77), d'autre part celle d'une matrice consonantique [k_d_l] : « [Comme de l] ongs échos [qui de 1] oin se [confond] ]ent", les deux réalisant la répétition signifiée par « écho ». Mais on peut aller plus loin si l'on se souvient que ce poème est le portrait d'une femme qui, outre par son interaction avec « Je » dans le huitain, se définit essentiellement par ses attributs sonores dans le sizain (la sonorité de son nom, de sa voix). Or si l'on considère les propriétés attribuées à la femme au vers 4 , on lit que celle-ci n'est « ni tout à fait la même, ni tout à fait une autre ». On peut ainsi prédiquer de la forme expressive identifiée exactement les mêmes propriétés que de la femme en question, puisque la 
forme dégagée l'est à chaque fois sur un fond de différences (la forme expressive est ainsi littéralement "ni tout à fait la même, ni tout à fait une autre »). Le rapport du «Je " à la femme de son rêve est ainsi le même que celui du lecteur à la forme expressive, qui iconise sur la modalité sonore les propriétés de la femme rêvée : le texte, en motivant l'identification femme/poème, se présente alors comme une sorte de blason sonore. Évidemment, cette sémiotisation de la matrice consonantique reste un phénomène affleurant momentanément dans un parcours interprétatif, que l'on ne saurait comparer à la routinisation sociale des acceptions lexicales. Mais cette différence est peut-être simplement de degré, et l'existence même de tels phénomènes a des conséquences théoriques importantes. Ils impliquent en particulier que l'«hiatus» entre modes sémiotique et sémantique, tel qu'il est formulé par É. Benveniste, parait inacceptable car faisant la part trop belle à une conception logico-pragmatique du mode sémantique pour laquelle celui-ci se caractérise essentiellement par ses aspects "référentielsintentionnels ${ }^{22}$. Or en réduisant le mode sémantique à cet aspect thétique-pragmatique du langage (référer à des entités, les mettre en relation, sous-entendre ou présupposer des contenus sur la base des contenus assertés, etc.), on se prive de la possibilité de décrire ce qui continue d'exister du mode sémiotique dans l'activité de parole et d'y repérer la manière dont ce que ce mode y trame affecte la fonction référentielle du langage. Ainsi du langage poétique, dont É. Benveniste constate à longueur de feuillets dans ses notes sur C. Baudelaire ${ }^{23}$ que son enjeu fondamental n'est pas celui de la référentialité, mais sans pour autant parvenir à la conclusion qu'une description adéquate de son fonctionnement aurait impliqué de décloisonner modes sémiotique et sémantique, à défaut de quoi on se voit réduit à insulariser le langage poétique, alors qu'il faudrait le voir comme déployant maximalement les différentes phases de l'activité langagière habituellement résorbées dans les usages « utilitaires ${ }^{24}$.

Nous conclurons en prolongeant l'examen de certains aspects des relations entre modes sémiotique et sémantique dans le cadre d'une discussion des propositions d'H. Meschonnic relatives à la notion de signifiance qu'il a contribué à développer.

\section{Conclusion : sémantique avec sémiotiques}

33 S'inscrivant explicitement dans la continuation d'É. Benveniste, H. Meschonnic (1997) a promu une énergétique du discours d'inspiration humboldtienne dont les dimensions référentielles-intentionnelles ne sont qu'un aspect, au demeurant souvent secondaire. Avec le couple conceptuel rythme/signifiance, l'objectif de l'auteur est de rendre possible la description du continu du discours (vs le discontinu du signe) et de la singularité de la signifiance d'un discours (vs la généralité de la signification) :

Je définis le rythme dans le langage comme l'organisation des marques par lesquelles les signifiants [...] produisent une sémantique spécifique, distincte $d u$ sens lexical, et que j'appelle la signifiance : c'est-à-dire les valeurs propres à un discours et à un seul. Ces marques peuvent se situer à tous les niveaux du langage : accentuelles, prosodiques, lexicales, syntaxiques. Contre la réduction courante $\mathrm{du}$ sens au lexical, la signifiance est de tout le discours, elle est dans chaque consonne, dans chaque voyelle ${ }^{25}$ qui, en tant que paradigme et que syntagmatique, dégage des séries [...]. Le sens n'est plus dans les mots. [...] Les mots ne sont que des passages du sens. (Meschonnic, 1982, p. 217/259). 
'auteur critique avec constance et virulence le concept de signe hérité de la philosophie $\mathrm{du}$ langage, inadéquat pour décrire la sémantique spécifique du discours (i.e.: la signifiance) en plaidant pour une « sémantique sans sémiotique » :

Quand il y a sémantique sans sémiotique [...] cesse alors la double articulation $\mathrm{du}$ langage. Car l'articulation en monèmes et en phonèmes, décrite par Martinet, n'est pertinente que dans les limites où le langage est pensé dans les termes de la langue, qui sont les termes du discontinu traditionnel entre les mots, entre les phrases, entre le son et le sens, ou la forme et le sens. [...] Dès que le langage est considéré dans l'ordre du discours, dans la physique du discours (et non plus seulement selon le logicisme de la pragmatique), et spécialement dans des systèmes de discours qui sont seuls l'accomplissement maximal du sémantique sans sémiotique, ce qui s'observe est le fonctionnement du continu, que masque la représentation du signe. (Meschonnic, 1997, p. 16).

(i) L'extension du domaine du sémantique par rapport à sa conception benvenistienne, le sémantique ne s'épuisant plus dans l'établissement d'une référence intentée, et dans une sortie du linguistique vers son extérieur, mais au contraire devant se lire aussi, voire plutôt, comme l'inscription d'un sujet dans la langue, dans un procès de subjectivation qui appelle une écoute particulière, qu'H. Meschonnic appelle le récitatif. De ce point de vue, si le mode sémiotique ressortit à ce qu'il peut y avoir de commun dans la langue, le mode sémantique concerne un procès de singularisation (de degré très variable) dans lequel se (re-)crée la langue en même temps que se pose un sujet du discours. Ainsi est soulignée, dans une perspective explicitement humboldtienne, la centralité de l'enérgeia langagière : le discours est avant tout activité, pour employer des termes cosériens, de (re)-création de la langue en même temps que d'invention d'un sujet.

(ii) Des propositions linguistiquement consistantes pour décrire avec précision des qualités prosodiques/accentuelles du signifiant textuel, envisagé dans son caractère continu, ce que les théories du texte issues du structuralisme ont rarement eu le souci de proposer. De ce point de vue, les propositions d'H. Meschonnic ont l'intérêt de mettre l'accent sur la dépendance de l'abstrait commun sémiotique par rapport au concret singulier sémantique, même si l'on peut regretter que sa problématisation se borne la plupart du temps à les installer dans un face à face irréductible, dans lequel la singularité subjective prime invariablement la régularité objective, ce pour quoi nous ne le suivrons pas jusqu'au bout. Dans les termes de la synthèse que nous avons proposée supra (Signification et signifiance : actuel déterminé vs virtuel indéterminé), on dira en effet que la signifiance alléguée relève de ce que nous avons dénommé signifiance 3, l'anathème antisémiotique empêchant cependant de décrire des effets mettant en jeu les autres relations (signification, signifiances 1 et 2), celles-ci présupposant, à un titre ou à un autre, le recours à la notion de signe. Un argument en notre faveur peut se lire dans les tensions dans l'œuvre meschonnicienne-même entre un positionnement théorique qui s'est tendanciellement durci à partir de Critique du rythme (Meschonnic, 1982), et des études de cas dans lesquelles on constate une approche plus nuancée, qui évoque explicitement la nécessaire articulation entre perspectives de la signifiance et de la signification. L'analyse de Chant d'automne (Meschonnic, 1973, p. 277-336) est exemplaire de cette tension. À propos du langage poétique, l'auteur note ainsi au début du texte : « tout y est signifiance, et rapport entre signifiance et signification. [...] Penser du sens faisait voir des formes. On ne commet pas ici l'inverse, qui serait une pseudo-omission de la signification, qu'on 
prétendrait ensuite retrouver par des "formes". On essaie plutôt d'étudier comment le poème fait signe. » (ibid., p. 286/297, nous soulignons) ou encore : « En tant que série dans

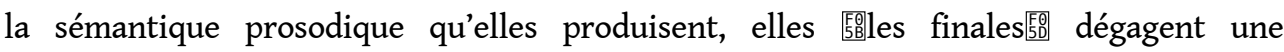
signifiance (production de sens à partir du signifiant) inséparable de la signification des mots-dans-leur-phrase dont elles sont les finales » (ibid., p. 294). Et dans ce passage, à propos de la sémantisation iconique d'une série lexicale où la dernière phrase ( «il n'y a pas de motivation après coup... ») apparait comme une dénégation du fait que l'analyse, avec la qualification "énonciation du cri ", avait excédé la description de la signifiance pour entrer dans celle de la signification :

La masse sémantique consonantique des continues et surtout des $/ \mathrm{R} /$, de frère à trop tard se rassemble comme cri et énonciation du cri, et devenir de l'intériorisation (profondes, languir, lourds, souvenir, remords, désespoir, avec les répétitions de cœur, de mort et de noir déjà indiquées). Il n'y a pas de motivation après coup, ni de psychologisation d'un / $/$ / en l'air, mais une organisation du langage par les signifiants. (ibid.).

On trouve d'autres exemples d'«iconicisation » dans le texte: "Cette chaîne situe le poème dans la prosodie des clausules de Spleen et idéal. Les finales de mots prédominent: les allongeantes, qui sont ici l'énonciation en acte du cri. » (ibid., p. 304, nous soulignons) ; « bercé et par ce sont liés par une répétition dans un entour vocalique (choc monotone) qui fait ce qu'il dit. » (ibid., nous soulignons). Ces exemples montrent, selon nous, que le monisme de la "sémantique du continu » invoqué face au dualisme sémiotique n'est pas tenable lors de descriptions effectives de textes, lors desquelles il apparait difficile sinon impossible de ne pas évoquer les effets de signification produits, qu'ils concernent, comme ici, des iconicisations de passages textuels, ou bien, plus fréquemment, les effets sémantiques produits sur les signifiés des unités lexicales mises en série. Les conséquences pour une théorie de la signifiance qui refuserait de se donner les outils conceptuels permettant de décrire ces phénomènes, ce que nous semble être celle défendue par H. Meschonnic dans ses formulations sémiophobiques les plus radicales, résident alors dans le constat d'une indicibilité théorique du sens impliquant que le mieux que l'on puisse faire pour décrire un texte soit de se mettre à son écoute. Mais après ? Si cette expérience est irremplaçable, elle n'est pas suffisante, et il demeure nécessaire d'articuler théoriquement continuité et discontinuité sémantiques, ce qui peut se faire notamment dans les termes des relations de signifiance et de signification telles que nous les avons discutées ci-dessus. Plutôt que défendre une "sémantique sans sémiotique ", nous plaiderions donc volontiers pour une "sémantique avec sémiotiques", qui requiert encore un effort d'élaboration conceptuelle pour dépasser le modèle du signe ${ }^{26}$ hérité de la philosophie du langage et décrire la diversité des régimes sémiotiques à l'œuvre dans l'activité langagière (Missire, 2018b). Dans cette perspective, l'étude du langage poétique, en tant que celui-ci actualise les potentialités générales du langage et requiert conséquemment de son lecteur une attention à toute la diversité des phases de la signifiance linguistique, est un soutien inappréciable.

NB : j'ai plaisir à remercier M. Favriaud pour ses remarques sur une première version de ce texte. 


\section{BIBLIOGRAPHIE}

BARTHES, R. (1974). « Théorie du texte ». Encyclopedia Universalis.

BENVENISTE, É. (1966). Problèmes de linguistique générale 1. Paris : Gallimard.

BENVENISTE, É. (1974). Problèmes de linguistique générale 2. Paris : Gallimard.

BENVENISTE, É. (2011). Baudelaire. Limoges : Lambert-Lucas.

COSERIU, E. (1952). « Sistema, norma y habla ». In : Teoría del lenguaje y lingüística general, cinco

estudios. Madrid : Gredos.

COSERIU, E. (2001). L'homme et son langage. Louvain-Paris : Peeters.

COSERIU, E. (2018). « Thèses sur le thème "langage et poésie" ». Pratiques 179-180.

DEssons, G. \& MEschonnic, H. (1998). Traité du rythme. Des vers et des proses. Paris : Dunod.

GÉRARD, C. (2018). «Variabilité du langage et productivité lexicale. Problèmes et propositions méthodologiques ». Neologica 12, p. 23-45.

GREIMAS, A. J. \& COURTÈS, J. (1993) [1979]. Sémiotique. Dictionnaire raisonné de la théorie du langage.

Paris : Hachette supérieur.

JENNY, L. (1990). La parole singulière. Paris : Belin.

KRISTEVA, J. (1969). Sèméiotikè. Recherches pour une sémanalyse. Paris : Éd. Le Seuil.

LACAN, J. (1966). «L'instance de la lettre dans l'inconscient ou la raison depuis Freud ». In: Lacan, J., Écrits 1. Paris : Éd. Le Seuil, p. 490-526.

LYOTARD, J.-F. (1971). Discours, figure. Paris : Klincksieck.

MESCHONNIC, H. (1973). Pour la poétique III. Une parole écriture. Paris : Gallimard.

MESCHONNIC, H. (1982). Critique du rythme. Anthropologie historique du langage. Lagrasse : Verdier.

MESCHONNIC, H. (1997). «Benveniste : sémantique sans sémiotique ». Linx 9, p. 307-326. En ligne : https://journals.openedition.org/linx/1075.

MEschonNic, H. (2006). Et la terre coule. Orbey : Arfuyen.

MISSIRE, R. (2014). « Sémiosis textuelle, stratification du champ attentionnel et déstratification des plans du langage ». In : Ablali, D., Badir, S. \& Ducard, D. (éds), Documents, textes, œuvres. Perspectives sémiotiques. Rennes : Presses universitaires de Rennes, p. 351-366.

MISSIRE, R. (2018a). « Unités linguistiques d'une sémantique discursive ». Langages 210, (2), p. 17-34.

MISSIRE, R. (2018b). « Flux psychique, sémiosis langagière et niveaux de l'analyse linguistique ». Signifiances (Signifying) 2, p. 207-225.

RASTIER, F. (2001). Arts et sciences du texte. Paris : Presses universitaires de France.

RASTIER, F. (2007). « Passages ». Corpus (6), p. 25-54. En ligne : https://journals.openedition.org/ corpus/832. 
RASTIER, F. (2017). « Cassirer et la création du structuralisme ». Texto ! 22 (4), p. 1-15. En ligne :

http://www.revue-texto.net/index.php?id=3977.

SAUSSURE, F. de (2002). Écrits de linguistique générale. Paris : Gallimard.

\section{NOTES}

1. C'est par exemple la définition d'É. Benveniste (1974, p. 51) : « Le caractère commun à tous les systèmes et le critère de leur appartenance à la sémiologie est leur propriété de signifier ou SIGNIFIANCE [...] »

2. «Dès les plus anciens textes, on relève des substantifs d'action tirés de participes présents, formation héritée du latin -(antia). » (TLF, entrée -ance).

3. « Le fait pour une unité de signifier » : la formulation a toujours déjà tendance à faire glisser le sens de « unité » vers le signifiant de celle-ci (on acceptera sans sourciller un énoncé comme « le signifiant X signifie que », plus difficilement « le signifié X signifie que »).

4. Dans «L'instance de la lettre dans l'inconscient ou la raison depuis Freud », dont la première publication remonte à 1957, J. Lacan (1966, p. 494-499) a en effet des formulations tout à fait explicites relativement à l'antécédence du signifiant par rapport au signifié, par exemple : "C'est là ce qui rendra possible une étude exacte des liaisons propres au signifiant et de l'ampleur de leur fonction dans la genèse du signifié. [...] Seules les corrélations du signifiant au signifiant y donnent l'étalon de toute recherche de signification. [...] Car le signifiant de sa nature anticipe toujours sur le sens en déployant en quelque sorte au devant de lui sa dimension. »

5. Dans la sémiotique greimassienne par exemple, le signifiant n'intervient qu'à la toute fin d'un parcours génératif entièrement situé sur le plan du signifié, la perspective générativeonomasiologique mise en œuvre secondarisant la matérialité langagière.

6. Par ailleurs largement fantasmé, cf. par exemple F. Rastier (2017).

7. On relève par exemple 28 occurrences du morphème travail- dans les 10 pages de l'article de R. Barthes cité supra.

8. Ou encore : «L'expressif est débordé par quelque chose que je ne peux qu'appeler un effet de signifiance, qui neutralise toute intentionnalité, tout problème de conscience ou d'inconscient [... ]. » (Meschonnic, 1982, p. 270).

9. Nous avons proposé de distinguer quatre régimes sémiotiques fondamentaux à l'œuvre dans la parole : signifiance, signification, expression, désignation (cf. Missire, 2018b).

10. "Il s'agit de savoir si et comment du signe on peut passer à la "parole". En réalité le monde du signe est clos. Du signe à la phrase il n'y a pas de transition, ni par syntagmation, ni autrement. Un hiatus les sépare. Il faut dès lors admettre que la langue comporte deux domaines distincts, donc chacun demande son propre appareil conceptuel. Pour celui que nous appelons sémiotique, la théorie saussurienne du signe linguistique servira de base à la recherche. Le domaine sémantique, par contre, doit être reconnu comme séparé. Il aura besoin d'un appareil nouveau de concepts et de définitions. » (Benveniste, 1974, p. 65).

11. Pour une approche de ces phénomènes dans un cadre théorique proche du nôtre, cf. Gérard, 2018.

12. Cela amène à nuancer la fameuse formule par laquelle É. Benveniste $(1966$, p. 131) clôt son article sur les niveaux de l'analyse linguistique: «nihil est in lingua quod non prius fuerit in oratione » : au niveau du système existe virtuellement un ensemble indéfini de combinaisons que l'usage n'a pas (encore) réalisé.

13. Texte entendu ici au sens de F. Rastier (2001, p. 21) comme « suite linguistique empiriquement attestée, produite dans une pratique sociale déterminée et fixée sur un support quelconque ». 
14. Tout cela dit bien trop rapidement. Nous avons développé plus amplement ces questions dans deux articles récents auxquels nous renvoyons le lecteur intéressé (Missire, 2018a ; 2018b).

15. Cf. supra Signifiance et signification: faire sens vs avoir un sens. La relation de conversion immédiate forme-sens au niveau de la relation de signification.

16. Dans la sémantique des textes de F. Rastier, c'est le concept d'interprétant qui permet de rendre compte de cette fonction de signifiance 3.

17. Ces éléments de description ont pour seule fonction d'illustrer le propos, ce qui explique leur caractère très fragmentaire.

18. De tels phénomènes de sommation (et de diffusion) s'observent sur les deux plans du signifiant et du signifié. Pour d'autres exemples, cf. F. Rastier (2007).

19. Chargé, dirait-on, en filant la métaphore électrique.

20. Je remercie M. Ballabriga d'avoir attiré mon attention sur cette assimilation.

21. Nous reprenons dans la suite du paragraphe quasiment à l'identique l'analyse présentée dans

R. Missire (2014).

22. Un exemple parmi d'autres : «Or l'expression sémantique par excellence est la phrase. [...] Il ne s'agit plus, cette fois, du signifié du signe, mais de ce qu'on peut appeler l'intenté, de ce que le locuteur veut dire, de l'actualisation linguistique de sa pensée. » (Benveniste, 1974, p. 222-223).

23. Cf. notamment aux pages : $28,42,50,62,124,130,132,136,384,398,426,430,442,448,540$, $554,558,554,634,638,644,656,658,668,718$. (Benveniste, 2011).

24. Cf. notamment les thèses 5, 6, 8 et 9 d'E. Coseriu sur le langage et la poésie (ici-même).

25. Le thème de la déstratification des niveaux linguistiques et de leur remobilisation à des fins de signifiance est commun aux auteurs ayant utilisé la notion : « La signifiance, qui est le texte au travail, ne reconnait pas les domaines imposés par les sciences du langage [...]; la signifiance lueur, fulguration imprévisible des infinis de langage - est indistinctement à tous les niveaux de l'œuvre : dans les sons, qui ne sont plus alors considérés comme des unités propres à déterminer le sens (phonèmes) mais comme des mouvements pulsionnels; dans les monèmes [...]; dans les syntagmes, dont importe, plus que le sens légal, la frappe, la résonance intertextuelle; dans le discours enfin, dont la "lisibilité" est ou débordée ou doublée par une pluralité de logiques autres que la simple logique prédicative. » (Barthes, 1974, p. 7). Il faudrait à cet égard mentionner aussi l'élaboration par L. Jenny (1990) de la notion de figural, qui est sur bien des aspects tout à fait affine aux réflexions sur la signifiance évoquées ici, retravaillées par l'auteur dans une perspective phénoménologique dans la lignée du Discours, figure de J.-F. Lyotard (1971).

26. Dans le cadre de la sémantique textuelle, F. Rastier a de longue date élaboré des propositions en ce sens (cf. par exemple Rastier, 2001).

\section{RÉSUMÉS}

Nous considérons dans cette étude la notion de signifiance telle qu'elle a été élaborée depuis les années 1960, en faisant l'hypothèse que, en dépit de la diversité des appropriations qui ont pu en être faites, celle-ci solidarise un ensemble de phénomènes fondamentaux pour l'étude du langage poétique. Le propos se veut de repérage et de synthèse : l'enjeu est de parvenir à dégager des points d'articulation entre considérations linguistiques et réflexions sur la signifiance issues des théories du texte, en identifiant quelques thèmes récurrents et en formulant des distinctions. Nous procédons en cinq temps : la première partie traite du couple signifiance/signification à la 
lumière de la distinction activité/produit dans le domaine langagier. La seconde déplace légèrement le point de vue dans la perspective de l'axe actuel/virtuel. La troisième introduit, sur la base des deux discussions précédentes, une distinction entre trois sous-types de signifiance, qui sont brièvement illustrées dans la quatrième partie. Nous proposons enfin en conclusion une discussion sur l'opposition benvenistienne du sémantique et du sémiotique ainsi que sa lecture meschonicienne.

We consider in this study the notion of significance as it has been developped since the 1960's in linguistics and text theories. We assume that this notion gives access to fundamental functionments of poetic language. We aim to submit a synthesis, and identify key points of articulation between fundamental semiolinguistics functionment and text theories. First we analyse the linked notions of significance and signification with the category activity/product in language, then with the category virtual/actual. We further introduce a distinction between three types of significances, that are finally illustrated. We conclude with a discussion on the

É. Benveniste's distinction of semiotics and semantics modes and its interpretation and developpements in H. Meschonnic's work.

\section{INDEX}

Mots-clés : signifiance, signification, langage poétique, sémiotique, semantics, Benveniste (Émile), Meschonnic (Henri)

Keywords : significance, signification, poetic languages, semiotics, semantics, Benveniste (Émile), Meschonnic (Henri)

\section{AUTEUR}

\section{RÉGIS MISSIRE}

Université Toulouse Jean Jaurès, F-31000, France 